\title{
LA EDUCACIÓN SUPERIOR EN URUGUAY
}

\author{
Cristina Contera*
}

* Doctora en Educación. Especialización en temas relacionados con la evaluación de instituciones de educación superior. Coautora de Pedagogía Universitaria. IESALC-UNESCO, 2001. Coordinadora de la Cátedra UNESCO Nuevas técnicas de enseñanza e innovación tecnológica en la educación superior, de la Asociación de Universidades GRUPO MONTEVIDEO (AUGM). Ha ocupado cargos de gestión académica en la Universidad de la República (UR). Desarrolla docencia de postgrado en Maestrías nacionales y en universidades de la región. Ha publicado trabajos sobre temas de su especialidad en libros y revistas de la región y Europa. Ha realizado Asesorías y Consultorías nacionales e internacionales en temas relacionados con la Evaluación Institucional. Actualmente es la Coordinadora Académica de la Comisión Sectorial de Enseñanza, Pro Rectorado de Enseñanza (UR) Además se ocupa de la Coordinación del Comité Académico de la AUGM denominado "Tecnologías de la Información y la Comunicación en Educación Superior" y el Proyecto "Generalización del uso educativo de las TIC en la UR" radicado en el Pro Rectorado de Enseñanza de la UR. Email: ccontera@oce.edu.uy

Resumen: La autora hace un análisis histórico de la Educación terciaria en Uruguay a través de los logros y reveses de sus legislaciones. Presenta informaciones generales acerca del sistema, los lineamientos generales de las reformas y los principales desafíos de la Es uruguaya.

Palabras claves: Educación Superior. Uruguay. Legislación. Reformas. Desafíos de la ES uruguaya.

Abstract: The author makes a historical analysis of tertiary education in Uruguay through the achievements and setbacks of its legislation. She presents general information about the system, the general framework of reforms and the major challenges faced by Uruguayan higher education.

Keywords: Higher education; Uruguay. Legislation. Reforms. Challenges of Uruguayan higher education.

\section{Descripción General}

En Uruguay existen actualmente cinco universidades oficialmente reconocidas, una pública, la Universidad de la República (UR) y cuatro privadas: la Universidad Católica del Uruguay (UCU), Universidad ORT, Universidad de Montevideo (UM), y Universidad de la Empresa (UDE) y 12 Institutos Universitarios.

De acuerdo a datos del año 2006, el número de alumnos matriculados en el sistema de educación terciaria ${ }^{1}$ público y privado uruguayo asciende a 121.775 alumnos en el ámbito de la enseñanza de grado, de los cuales $\mathbf{1 3 . 5 9 3}$ de ellos pertenecen al sistema privado (11\% del total) y 81.774 se agrupan en la Universidad de la República ( $89 \%$ del total $)^{2}$

De acuerdo a su Ley orgánica (Ley 12549/58) la UR es una persona jurídica pública, que funciona como Ente Autónomo. Por esta razón no necesita una autorización expresa de parte de un poder central para modificar su estructura, crear nuevas carreras, modificar planes, contratar docentes, etc.

Hasta el año 1984, en que se crea la primera Universidad Privada de carácter confesional -la Universidad Católica del Uruguay-, la UR detenta el

1 La educación terciaria en Uruguay comprende a la educación superior: Universidad pública y Universidades e Institutos Universitarios privados, y un grupo heterogéneo de ofertas de educación terciaria no universitaria constituido por Institutos de Formación Docente, cursos técnicos dependientes del Consejo de Educación Técnico-Profesional y Escuelas y Centros dependientes de Ministerios.

2 Datos construidos a partir de cifras extraídas del "Anuario Estadístico de Educación 2006" Ministerio de Educación y Cultura (MEC) www.mec.gub.uy y datos preliminares del Censo 2006 de Estudiantes, U.R. 
monopolio de la educación superior en el país. El decreto-Ley $\mathrm{N}^{\mathrm{0}} 15661 \mathrm{del}$ 20 de noviembre de 1984 "Universidades Privadas", establece en su Artículo 1 que "Los títulos profesionales que otorguen las Universidades Privadas, cuyo funcionamiento haya sido autorizado por el Poder Ejecutivo, para su validez deberán ser registrados ante el Ministerio de Educación y Cultura, que organizará el Registro correspondiente"

Posteriormente a la creación de la UCU, se decreta un sistema de regulación de la oferta privada de Educación Superior. El decreto 308/95 del 11 de agosto de 1995, establece un Ordenamiento del Sistema de Enseñanza Terciaria Privada gestionado por el Ministerio de Educación y Cultura (MEC) de Uruguay. En los fundamentos se señala que "en vistas de que comienzan a proliferar instituciones privadas que ofrecen públicamente enseñanza post-secundaria, se hace aconsejable establecer un régimen que permita otorgar un reconocimiento oficial de calidad a aquellas que acrediten ante el Ministerio de Educación y Cultura el adecuado nivel académico de la enseñanza impartida y los títulos que expidan" (Decreto 308/995, pág. 6)

En la Ley Nro 16.736 del 5 de enero de 1996 se señala en su Artículo 380 que "Se reserva el uso de la denominación Universidad o sus derivados, así como atribuir carácter "superior" a la enseñanza que impartan y aplicar a los títulos y certificados que expidan las denominaciones "licenciatura", "maestría", "magíster" y "doctor", o sus derivados, a las instituciones privadas cuyo funcionamiento hubiera sido autorizado de conformidad con las normas vigentes".

El sistema de Educación Universitaria uruguayo está regulado por la Ley Orgánica Universitaria, Ley 12549 del 29 de octubre de 1958, el Decreto Ley Nro 15661 del 20 de noviembre de 1984 referido a las universidades privadas, el Decreto-Ley No 308/995 aprobado el 11 de agosto de 1995 y la Ley Nro 16736 del 5 de enero de 1996 del Presupuesto Nacional de Sueldos Gastos e Inversiones.

El sistema de educación terciaria no universitaria de carácter público, está constituido por una red nacional de centros de formación de docentes para los subsistemas primarios, medio y técnico. Su matrícula supera los 20.000 alumnos, según informaciones del Ministerio de Educación y Cultura del año 2005. El sistema de educación terciaria no universitaria es parte integrante del Ente Autónomo denominado "Administración Nacional de Educación Pública" (ANEP) creado por Ley 15.739 promulgada el 25 de marzo de 1985. Dicho Ente agrupa los subsistemas de educación primaria, media, técnico-profesional y la formación de docentes. 
En Uruguay el proceso de privatización de la Educación Superior es reciente y su desarrollo incipiente. Esta afirmación está sustentada en los siguientes indicadores:

a) En el período 1984-2007 se crean las Universidades e Institutos Universitarios.

b) Esta oferta privada de educación superior se limita a cuatro Universidades, tres laicas y una confesional y doce Institutos universitarios.

c) La matrícula privada global de grado representa un $11 \%$ del total de matriculados en el sistema de educación superior.

Por último es importante mencionar que, siendo Uruguay un país que no exige habilitación previa al funcionamiento de las instituciones educativas privadas, dado que existe el principio de "Libertad de Enseñanza" presente en la Constitución de la República, resulta imprescindible, desde la perspectiva universitaria, limitar la denominación de "Universidad" a aquellas instituciones que ofrezcan garantías de calidad y pertinencia de la oferta. Se pone especial atención a la existencia de requisitos mínimos asociados a la complejidad de las funciones a cumplir, la calidad del personal académico y administrativo, el estilo de gobierno, etc.

\section{Historia y contexto nacional y regional}

\subsection{Breve historia}

Siguiendo el modelo de Universidad napoleónica; el Reglamento Orgánico de octubre de 1849 creaba una Universidad que comprendía la totalidad de la instrucción pública: primaria, secundaria y superior.

El espiritualismo ecléctico - como corriente filosófica - predominó en nuestra Universidad desde su fundación; y sirvió como fundamento de la línea anticlerical y liberal que caracterizó a la Universidad hasta 1885. Según Ardao (1968) "Desde la instalación de la Universidad, en 1849, quedó consagrada la influencia exclusiva y directa de Francia, bajo la forma del espiritualismo ecléctico de la escuela de Cousin, que imperó incontrastable durante el tercer cuarto de siglo pasado. Fue seguida, en el último cuarto del mismo -aunque sin desplazar del todo, desde luego, a la francesa - por la de Inglaterra, al imponerse el positivismo en su modalidad sajona, con los nombres de Darwin, Spencer, Stuart Mill y Bain al frente" (Ardao,1968:8)

El nacimiento de la universidad moderna se corresponde con el período en que la Universidad procesa su segunda estructura institucional; determinada por 
la ley orgánica de julio de 1885 que consagraba una moderada descentralización administrativa respecto del Poder Ejecutivo. Desde 1880 Alfredo Vásquez Acevedo -hombre que adhería al positivismo- desde el rectorado, llevó adelante una vasta y sustancial reforma de la enseñanza universitaria.

La Reforma de 1885 "reorganizó el gobierno de la universidad, suprimió la libertad de estudios en la enseñanza superior, ajustó la distribución de Facultades, reglamentó la expedición de títulos, para citar sólo algunos de sus aspectos. Fue, sin embargo, al margen de ella, a través de la gestión administrativa de las autoridades universitarias, que la parte concreta y sustantiva de la reforma se cumplió, transformando de arriba abajo el cuerpo y el espíritu de la casa de estudios." (Ardao,1950:.67)

Dicha reforma dio nacimiento a la universidad moderna introduciendo criterios organizativos que perseguían mayor eficiencia y ejecutividad; y sintonizó a la enseñanza universitaria con el espíritu científico positivista de la época.

La etapa comprendida entre los años 1908 y 1917 conocida como "La Reforma" o "La Reorganización" comprende la tercera estructura institucional de la existencia universitaria. Bajo la vigencia de la ley orgánica de 1908 se descentralizó y dio autonomía técnica al gobierno de las Facultades.

Las primeras décadas del siglo XX fueron conmovidas por las movilizaciones de carácter reformista; movilizaciones que contaron con el protagonismo estudiantil inspirado en el estallido de 1918 de la Universidad de Córdoba.

En nuestro país, los estudiantes reclamaron su participación en los temas más sustantivos y permanentes. Diversas concepciones se enfrentaron en el seno de la Universidad; se discutía sobre la orientación social de la enseñanza, la renovación pedagógica, la autonomía y sobre una concepción de gobierno universitario. El movimiento renovador estudiantil luchó intensamente por transformar a la Universidad en un centro cultural comprometido con los problemas nacionales, autónomo respecto del poder político y proyectado hacia los sectores sociales marginados por la educación.

El movimiento estudiantil condujo al nacimiento en 1929 de la Federación de Estudiantes Universitarios (FEUU), a la creación de su órgano "Jornada", y a la realización del Primer Congreso Nacional de Estudiantes, que centró sus discusiones en el tema de la Reforma Universitaria (1930).

Sin embargo, en marzo de 1934, la dictadura del Dr.Gabriel Terra promulgó una ley que reaccionando contra todas las aspiraciones reformistas y autonómicas sancionó una total sujeción administrativa de la Universidad al Gobierno Nacional. La respuesta se tradujo en huelgas, manifestaciones callejeras y en la reunión de una Asamblea del Claustro que elaboró el célebre Estatuto de 
1935, que aunque imposible de comenzar entonces a regir, transformaba los fines de la Universidad, reafirmaba su autonomía y proponía un régimen de co-gobierno entre docentes, estudiantes y egresados ${ }^{3}$.

Durante el período examinado, sucesivas leyes dictadas hasta 1945, multiplicaron el número de facultades, dándole a la Universidad su actual conformación.

La Constitución promulgada el 18 de mayo de 1934 mantuvo la autonomía universitaria que consagrara el Art.100 de la Constitución de 1917, y lo propio hizo la Constitución de 1942. Finalmente, la Constitución de 1952 amplió la autonomía universitaria disponiendo además que el Consejo Directivo de la Universidad "será designado por los órganos que la integran, y los Consejos de sus órganos serán electos por docentes, estudiantes y egresados" conforme a lo que debería establecer una Ley sancionada por mayoría absoluta del total de componentes de cada Cámara. Se consagró así, con rango constitucional, el co-gobierno de la Universidad por tres órdenes: docentes, estudiantes y egresados. Consultada la Universidad para elaborar su nueva ley orgánica -como lo mandaba la Constitución- el Consejo Directivo Central se expidió finalmente en abril de 1958, elevando a las cámaras el anteproyecto de la actual ley orgánica universitaria.

Hacia fines de la década del cuarenta, las principales figuras del liderazgo reformista asumieron la dirección de la estructura universitaria; una nueva Universidad comenzaba a emerger.

Fueron tiempos innovadores, en donde la Universidad estuvo abierta a la pluralidad de opiniones y atenta a los reclamos sociales así como al advenimiento de profundos cambios generados por la crisis económica. En ese período se manifestaron un conjunto de propuestas tendientes a lograr una paulatina transformación; el principio rector de esta etapa fue el de poner a la Universidad al servicio del pueblo, y del país todo. (Paris,B.,s/f)

Esta etapa se caracterizó por la ebullición permanente de ideas y proyectos que manifestaron la preocupación constante de incidir en la vida cultural y productiva del país desde una perspectiva latinoamericana. La comunidad académica implementó un conjunto investigaciones que proponían la descripción e interpretación de la realidad socio - económica nacional. Estos esfuerzos se implementaron de forma paralela al incremento de la matricula universitaria.

La ley orgánica de 1958 fue la culminación de los principios y las soluciones que se habían ido forjando tras una larga evolución histórica: autonomía

3 El Estatuto fue particularmente radical en la defensa del fuero universitario. Su art. 136 proclamaba: "Es obligación esencialísima de todo dirigente, funcionario u organismo la defensa del fuero universitario"... "incluso hasta la resistencia activa, contra todo desconocimiento y violación". 
universitaria: gobierno integrado con la participación de tres órdenes (docentes, egresados y estudiantes); principio de libertad de cátedra y de opinión; definición precisa de los fines de la Universidad.

La década del 60 presenció la profundización de la crisis económica y social gestada en los años $50 \mathrm{y}$, generó también en la comunidad universitaria una preocupación por redefinir el papel de la Universidad, que fue común a círculos universitarios de otros países latinoamericanos.

A partir del Golpe de Estado ocurrido el 27 de junio del año 1973, se disuelve el Parlamento (Poder Legislativo) y el país ingresa en un período conocido como "Dictadura". El peso de esa realidad incidió sobre la Universidad y todo el sistema educativo nacional. El estudiantado, sensibilizado con los movimientos del "68" ocurridos en Europa, Estados Unidos y América Latina, redobla sus reclamos frente al poder político y acompañan -a tono con las tensiones sociales reinantes- las reivindicaciones de los sectores sindicales más combativos o perjudicados. La Universidad es finalmente intervenida por el poder militar. Según las palabras de Ardao en carta enviada a Blanca París:

"La Universidad en que se ensañó la intervención, constituyó el desenlace histórico de un movimiento de reformismo incesante, iniciado en el país con anterioridad, aún, al episodio de Córdoba. Los protagonistas de éste, recabaron y utilizaron en forma expresa, para sus formulaciones de 1918, algunos antecedentes uruguayos" (Ardao, 1985:28)

La consecuencia de la intervención en la Universidad fue la paralización de la vida académica en todas sus facultades y escuelas que la componían. La totalidad de la estructura universitaria se vio afectada profundamente por la acción de la dictadura. "Puede hablarse, sin temor a exagerar, de la delimitación de la Universidad por parte de la dictadura uruguaya, en cuanto las funciones esenciales de una institución de enseñanza superior, le han sido cercenadas o suprimidas". (Maggiolo,1988:7)

Campodónico, Massera y Sala (1991) caracterizan el impacto de la dictadura uruguaya en la educación en término de exclusiones: Con relación a los docentes, con respecto al conocimiento y con referencia a los métodos. "El universo del conocimiento fue cerrado, altamente clasificado, fragmentado y esquematizado, ajeno al momento histórico, y a la realidad de la comunidad circundante y del país y al saber popular, que transmitió pautas de socialización esencialmente individualistas y falsamente meritocráticas" (Campodónico et al,1991:155) Y agregan con respecto al contenido ideológico de la dictadura: "Se despliega una visión del mundo regido por un Orden natural fijo, in- 
mutable, armónico, sin contradicciones y con un carácter claramente teológico". (Campodónico et al, 1991:91)

Después del histórico plebiscito de 1980, cuando el pueblo uruguayo contestó masivamente que no a la institucionalización del gobierno de facto, el Uruguay se encaminó hacia la restauración de la vida democrática. La movilización de todas las fuerzas políticas y gremiales fue ganando espacios hacia la concreción de las elecciones nacionales en noviembre de 1984. Los universitarios, concertaron con los grupos políticos y los gremios el retorno de la Universidad a la vida autónoma, ellos fueron los primeros en reorganizarse -cuando hacerlo era severamente penado- la suya fue la primera señal que afloró del renacimiento universitario. Simultáneamente, docentes y funcionarios comenzaron a reagruparse y se sucedieron instancias de reflexión colectiva sobre el papel de la Universidad en la difícil coyuntura del país.

Luego del período de intervención, la Universidad tomó diversas medidas que implicaron su total reestructuración. En marzo de 1985 la Universidad recuperó su autonomía, iniciando un período conocido como de reinstitucionalización.

A partir de la situación de desmantelamiento y decadencia en que reciben la Universidad, más que a elaborar un nuevo modelo al cual ajustarse, las autoridades legítimamente electas se proponen apoyar a los polos más activos en investigación, docencia, capacitación y extensión, con lo cual se traza sobre el camino una política de renovación científica y tecnológica, adecuando al mismo tiempo las carreras profesionales a las necesidades futuras del país.

A partir del año 1985, momento en que se retoma la vida democrática, la U.R. realiza importantes esfuerzos de recomposición de sus cuadros docentes y de la calidad en el cumplimiento de sus funciones. Según las "Memorias del Rectorado" del año 1998, en el período 1989-1998 se producen grandes transformaciones entre las que es posible mencionar: la modificación de más de 90 planes de estudio, la puesta en marcha de seis nuevas facultades e institutos asimilados a Facultad, la puesta en práctica de 20 nuevas carreras de grado, la implementación de 50 postgrados y especializaciones (doctorados, maestrías y diplomas de postgrado), la ejecución de 35 proyectos de extensión y actividades en el medio, la firma de más de 700 convenios con el sector productivo y de servicios, el incremento de las horas docentes en un $86 \%$ en los años 1986-1997, el incremento del porcentaje destinado a la investigación científica de $6 \%$ en 1985 a $20 \%$ en 1997, con un crecimiento exponencial en 
los cargos con régimen de Dedicación Total que pasaron del exiguo número de 37 en 1985 a 410 en el presente año(Brovetto, 1998:421-423) En la actualidad más del $80 \%$ de la producción científico-tecnológica del país, se lleva a cabo en la UR. El resto se encuentra distribuido en agencias estatales o paraestatales de investigación y en un escaso porcentaje en el sector productivo.

De estas iniciativas destacamos el Convenio para el Desarrollo de las Ciencias Básicas (PEDECIBA) firmado el 22 de octubre de 1986. Este Programa abarcó a las siguientes ciencias básicas: Biología, Física, Informática, Matemática y Química. Este Convenio se firma entre el Ministerio de Educación y Cultura (MEC) y la Universidad de la República (UR) En el documento se señala que: "El aporte de las ciencias básicas es válido en cualquier etapa de desarrollo pero resulta tanto más necesario cuando éste es incipiente o sufre retrasos debido a factores de índole económica o política. Para una sociedad que utiliza tecnología importada y que en ocasiones no está capacitada para adaptarla a las situaciones locales, fortalecer el estudio de las ciencias básicas es un paso imprescindible para reducir esa dependencia de factores externos" (Convenio PEDECIBA, 1986.Art.I,inciso B)

Según ese enfoque resulta claro que la Universidad para recuperarse y desarrollarse debe responder a la realidad socioeconómica del país, pero que no puede quedar pasivamente supeditada a ella: que su consolidación democrática y su dinámica docente depende de esa permanente interacción, pero sin olvidar que estamos inmersos en un nuevo universo de conocimientos frente a los cuales la Universidad no puede rezagarse; que se han operado cambios sustantivos en el entorno económico, político, social, donde se afronta un mundo mucho más competitivo; y por último, que las sociedades latinoamericanas -y desde luego la uruguaya con ellas- se han venido empobreciendo dramáticamente y sin tregua en esta década de los años ochenta. En este contexto, la Universidad estima esencial llevar a cabo una decidida política de apertura que, según conceptos del Rector Lichtensztejn, debe operar a través de cuatro grandes ejes: la apertura institucional, social, regional e internacional.

Y con respecto a los pilares de la Universidad que debían ser reafirmados señalaba el Rector: "Cuando definimos la necesidad de una nueva Universidad, la urgencia de una Universidad remozada y renovada, debemos reconocer en ese reclamo tres clases de objetivos. Unos son de reafirmación histórica de la Universidad Autónoma; otros de reafirmación o readecuación de elementos de la política universitaria y finalmente los aspectos efectivos de la innovación" (Lichtensztejn,1985:33) 


\subsection{Rasgos actuales de la U.R.}

La educación pública en Uruguay, ha recibido en las últimas décadas una dotación presupuestal en términos del porcentaje del PBI asignado, que es una de las más bajas de la región. Comparte con Honduras, República Dominicana, Guatemala, El Salvador y Haití, los últimos lugares correspondientes a los países que reciben menos del $3 \%$ del $\mathrm{PIBI}^{4}$

Esta tendencia se mantiene inalterada en las últimas dos décadas, y se acompaña de un crecimiento sostenido de su matrícula ${ }^{5}$. Hasta el año 2004, que marca el inicio de modificación de este estado de cosas, la U.R recibió un presupuesto con montos casi constantes, mientras la matrícula universitaria creció más del $40 \%$.

Las restricciones presupuestales impactan de forma significativa en los salarios docentes. Guarga (2007) señala al respecto que: "Este hecho muestra un alto compromiso institucional del personal docente y no docente de la institución que prioriza la atención a la demanda de incremento del alumnado aún a costa del deterioro de su salario y no se recurre al cobro de matrícula o al establecimiento de límites de ingreso, en los términos que desde algunos ámbitos políticos se pretendía. Esto es una afirmación de la pertinencia en lo que hace al acceso a la educación superior, decidida en el ámbito de la autonomía de gobierno de la institución. (Guarga, 2007:56)

Para el tramo 2006-2009 el gobierno que se instaló el 1 ro de marzo de 2005, aprueba un incremento del $50 \%$ del presupuesto universitario, en concordancia con el crecimiento del PBI previsto durante dicho período. Según datos del Anuario Estadístico 2006 del MEC, el gasto en educación consolida en el año 2006 la reversión del deterioro pronunciado del período 2002-2004. Y agrega. "Se identifica el comienzo de una recuperación que se manifiesta en el aumento de la inversión en educación en términos constantes y el acompañamiento del ritmo de crecimiento de la economía" (MEC, 2007:52)

Se consolida así, el fortalecimiento de una tendencia histórica en Uruguay:

4 Según datos del PNUD sobre Desarrollo Humano correspondiente al año 2006, Uruguay ocupa el lugar 117 respecto al gasto público educativo como porcentaje del PBI.

5 La tasa bruta de escolarización en Uruguay está algo por encima del $30 \%$. La tasa de la zona metropolitana y alrededores es del $41 \%$ En el interior del país esta tasa desciende al $16 \%$ ubicándose por debajo de la media Latinoamérica. Según datos de los Censos Estudiantiles la matrícula crece de manera constante en las últimas décadas: en 1960 el número de alumnos era de 15320; en 1968 se contabilizaban apenas 18.650 estudiantes. En el año 1988 esta cifra se había triplicado: 61.428. En la actualidad esta cifra asciende a 81.774 alumnos de los cuales un $63 \%$ son mujeres y un $37 \%$ son hombres. Un $56 \%$ de los estudiantes universitarios desempeñan actividades laborales remuneradas. (Datos preliminares del Censo 2006) La matrícula proyectada para el año 2030 según estudios realizados (Fernández et al, 2000:4) es de 172.000 estudiantes, considerando un crecimiento medio del ingreso y el clima educativo de los hogares. 


\section{la reafirmación del principio de gratuidad e ingreso irrestricto a la Uni- versidad pública.}

En lo que respecta a su estructura organizativa, la U.R. goza de una amplia autonomía, la que está garantizada por su Ley orgánica (Ley 12549) donde se expresa en su Artículo 1 que "La Universidad de la República es una persona jurídica pública, que funcionará como Ente Autónomo de acuerdo con las disposiciones pertinentes de la Constitución, esta Ley Orgánica y demás leyes y los reglamentos que la misma dicte".

En su artículo 2 se expresa con respecto a los FINES de la Universidad: "La Universidad tendrá a su cargo la enseñanza pública superior en todos los planos de la cultura, la enseñanza artística, la habilitación para el ejercicio de las profesiones científicas y el ejercicio de las demás funciones que la ley encomiende. Le incumbe asimismo, a través de todos sus órganos, en sus respectivas competencias, acrecentar, difundir y defender la cultura; impulsar y proteger la investigación científica y las actividades artísticas y contribuir al estudio de los problemas de interés general y propender a su comprensión pública: defender los valores morales y los principios de justicia, libertad, bienestar social, los derechos de la persona humana y la forma democrático-republicana de gobierno". Y agrega en su artículo 5 que: "La universidad se desenvolverá, en todos los aspectos de su actividad, con la más amplia autonomía”.

$\mathrm{Su}$ principal órgano de gobierno es el Consejo Directivo Central al que le compete la administración, dirección general de la Universidad y la superintendencia directiva, disciplinaria y económica sobre todas las Facultades, Institutos y Servicios que la componen.(Artículo 20)

Las atribuciones del Consejo Directivo Central, son: establecer la dirección general de los estudios universitarios, dirigir las relaciones de la Universidad, coordinar la investigación y la enseñanza impartida por las distintas Facultades y los demás Institutos y Servicios que constituyen la Universidad, aprobar los planes de estudio, establecer títulos y certificados, establecer las condiciones de admisión y certificados de estudios extranjeros, revalidar esos títulos y certificados, dictar los reglamentos necesarios para el cumplimiento de sus funciones, reglamentar las elecciones universitarias y efectuar las respectivas convocatorias, designar a todos los funcionarios docentes, técnicos, administrativos de servicio u otros de su dependencia, fijar las directivas generales para la preparación de proyectos de presupuestos, resolver la creación, fusión o división de facultades y declarar las asimilaciones de Institutos o Servicios a Facultad (Artículo 21)

La ley señala además de forma expresa que "La enseñanza universitaria oficial es gratuita. Los estudiantes que cursen sus estudios en las diversas de- 
pendencias de la UR no pagarán derechos de matrículas, exámenes ni ningún otro derecho universitario. Los títulos y certificados de estudio que otorgue la Universidad de la República se expedirán gratuitamente, libres del pago de todo derecho" (Artículo 66)

Actualmente la UR institución pública estatal abarca 27 servicios $^{6}$ (Facultades, Escuelas y Sedes del Interior) estimándose en cerca de 5949 los docentes, distribuidos en $7358 \operatorname{cargos}^{7}$. Ofrece más de 200 títulos o diplomas de pre grado, grado y postgrado. Desarrolla cursos de educación permanente para los graduados, así como de formación pedagógica. Estos últimos se llevan a cabo por los Servicios y las Áreas, a cargo fundamentalmente de Unidades de Apoyo a la Enseñanza (UAE) y cuentan con apoyo central de la Comisión Sectorial de Enseñanza (CSE)

La puesta en práctica de los postulados de su Misión ha contribuido a la conformación de un amplio margen de legitimación y aceptación social, al punto de que la mayoría de los ciudadanos de este país asocian esta Casa de Estudios con la adhesión y defensa de los intereses sociales, políticos y culturales más amplios. Asimismo, el estatuto de la gratuidad del servicio históricamente asumido y vigente en la actualidad, así como su estilo de gobierno participativo ${ }^{8}$ y la amplia autonomía de que dispone, han configurado una particular cultura académica que se aleja bastante de las actuales tendencias hacia la extrema selectividad ${ }^{9}$.

6 Los Servicios universitarios abarcan Facultades y Escuelas que se agrupan en 5 Áreas: Área Agraria: Facultad de Agronomía y Facultad de Veterinaria; Área Artística: Instituto Escuela Nacional de Bellas Artes y Escuela Universitaria de Música; Área Científico-Tecnológica: Facultad de Química, Facultad de Ingeniería, Facultad de Arquitectura, Facultad de Ciencias; Área Social: Facultad de Derecho, Facultad de Ciencias Sociales, Facultad de Ciencias Económicas, Facultad de Humanidades y Ciencias de la Educación; Escuela Universitaria de Bibliotecología y Ciencias Afines; Licenciatura en Ciencias de la Comunicación, Escuela de Administración; Área Salud: Facultades de Odontología, Medicina, Psicología Enfermería; Escuelas de Nutrición y Dietética, Parteras, Tecnología Médica, y Tecnología Odontológica. Sedes Universitarias del Interior del país: Centro Universitario de Paysandú, Centro Universitario de Rivera, Centro Universitario de Regional Este, Regional Norte-Sede Salto.

7 De acuerdo a los datos aportados por el II Censo Docente de la UR (1999), el número total de personas con cargos docentes remunerados hasta el 31 de mayo de 2000, incluidos becarios con perfil docente, asciende a 5949, de los cuales 3089 son hombres y 2851, mujeres. Un $43.9 \%$ del total declara poseer algún estudio de posgrado y un $20.4 \%$ se desarrolla tareas de investigación. El número de cargos es superior debido a que los docentes pueden acumular cargos dentro de la institución. Datos del año 2005 del Servicio Universitario de Información contabiliza 7643 cargos docentes, de los cuales apenas 1225 son Profesores Agregados Grado 4 y Titulares Grado 5. Solamente 524 docentes poseen Dedicación Exclusiva a la U.R.. (Fuente: Comisión Sectorial de Investigación Científica de la U.R., 2002)

8 El gobierno de la UR según lo especificado en su Ley Orgánica (Ley 12549 del año 1958) comprende la participación de estudiantes, docentes y egresados. Comúnmente se habla del co gobierno como del instituto jurídico por medio del cual se establece la co-gestión de docentes, estudiantes y egresados en los órganos del gobierno universitario tanto centrales como sectoriales.

9 Según lo especificado por Clyde Barrow (1996) la estrategia de la Selective Excellence (Excelencia Selectiva) implica básicamente la redefinición de la misión histórica de las Universidades y una articulación más estrecha con el gobierno y las demandas de la industria y el mercado. Esta estrategia encuentra eco en 
La inserción de Uruguay en el Mercado Común del Sur (MERCOSUR) tiene su origen en un Acuerdo Regional de Integración suscrito originalmente entre cuatro países: Argentina, Brasil, Paraguay y Uruguay, al que posteriormente se agregan dos más: Bolivia y Chile, en calidad de asociados (Tratado de Asunción, del 26 de marzo de 1991) ${ }^{10}$

\subsection{Integración regional y Redes Académicas}

La necesidad de iniciar un sistema de reconocimiento de los títulos profesionales, dirigido a facilitar la movilidad académica en la región, indujo a los Ministros de Educación de los cuatro países a crear un Grupo de Trabajo ad- hoc con el cometido específico de llevar a cabo esta tarea. El Grupo de Trabajo de Especialistas en Evaluación y Acreditación de la Educación Superior (GTEAE) fue creado con el cometido de "elaborar una propuesta de organización de un sistema de acreditación de instituciones y carreras, que permita facilitar el reconocimiento de sus títulos de grado" (Acta de la XII Reunión de Ministros de Educación. Asunción, 11/07/97)

En la V reunión del Grupo realizada en los días 27 al 29 de octubre de 1998, en la ciudad de Brasilia, se seleccionan las tres carreras que serán propuestas para la incorporación a la primera etapa de implementación del procedimiento de acreditación establecido en el "Memorándum de entendimiento sobre la implementación de un mecanismo experimental de acreditación de carreras para el reconocimiento de títulos de grado universitario en los países del Mercosur”. Dichas carreras son: Ingeniería, Medicina y Agronomía. En el momento actual dichas carreras han sido acreditadas y se encuentran en proceso de acreditación las Facultades de Veterinaria, Arquitectura, Enfermería y Odontología.

La expresión exclusivamente académica del proceso de integración regional adoptó la forma de Red Académica denominada "Asociación de Universidades Grupo Montevideo" (AUGM) Esta Asociación fue creada en 1991 e involucra en la actualidad a 21 universidades públicas ${ }^{11}$ con grado de desarrollo científico-tecnológico compatible, con idénticas problemáticas y análogos objetivos políticos. Según Jorge Brovetto (1998), la modalidad de cooperación subregional por el que opta la AUGM, es contrario al modelo de "cooperación vertical" ya que selecciona y aplica un estilo horizontal de cooperación e intercambio académico.

el sector de los académicos afines al lenguaje de la meritocracia quienes usan el espacio institucional para llevar a cabo una estrategia de gran productividad de acuerdo a los parámetros internacionales.

10 El "Tratado de Asunción" no constituye el MERCOSUR per se, sino el instrumento de carácter internacional destinado a hacer posible su concreción. (Art. 6)

11 Para más información ver: www.augm.edu.uy 
La finalidad principal de la AUGM es promover el desarrollo académico, científico y la capacidad tecnológica de los países de la región e impulsar el proceso de integración regional a través de la conformación de un espacio académico común ampliado, con base en la cooperación científica, tecnológica, educativa y cultural entre todos sus miembros. Se trata de la conformación de un sistema regional apto para aprovechar solidariamente, para el conjunto, los logros y ventajas de cada institución universitaria, contribuyendo con esto a crear, una masa crítica de personal altamente calificado; y encarar el estudio de problemas sub regionales comunes en áreas prioritarias del desarrollo social, la salud, el medio ambiente, la producción, la cultura, la educación, etc.

Distintos programas, con un alto grado de descentralización operan en este espacio compartido. Entre ellos podemos destacar los Núcleos Disciplinarios (ND), Comités Académicos, así como tres Cátedras UNESCO.

Los Núcleos disciplinarios son agrupamientos académico-técnicos correspondientes a una disciplina de interés común, donde cada miembro aporta sus disponibilidades tanto en personal de alta calificación como en recursos materiales, para actividades científicas, técnicas, docentes, de desarrollo, de extensión, etc. ${ }^{12}$ y están radicados en las Universidades que representan la máxima fortaleza e interés en el Área correspondiente.

Los Comités Académicos son organismos académico-técnicos concebidos para abordar, con enfoque multi e interdisciplinario, grandes configuraciones temáticas calificadas como estratégicas por comprender cuestiones amplias, de carácter regional más que nacional, que deben encararse conjuntamente con la oferta académica científico-técnica integrada de las Universidades del Grupo. Los temas que abordan estos Comités son: Medio Ambiente, Agua como Recurso Regional, Desarrollo Tecnológico Regional, Salud, Desarrollo Regional (Rural y Urbano), Ciencias Políticas y Sociales, Comité Académico de Producción Artística y Cultural.

La U.R. participa activamente desde la creación de la AUGM en los distintos Programas. Dicha inserción ha posibilitado el establecimiento de sólidos vínculos de colaboración e intercambio académico desde una perspectiva, plural y participativa y un enfoque cooperativo y solidario.

\subsection{Planes Estratégicos de Desarrollo de la UR (PLEDUR) 2000-2010}

En los años 2000 y 2005, la UR formula sus "Planes Estratégicos de Desarrollo" (PLEDUR) para los quinquenios 2000-2004; 2005-2009 ${ }^{13}$. Dichos

13 Para más información consultar el Portal de la U.R. www.universidad.edu.uy 
planes marcan el inicio de una etapa de ordenamiento de la planificación presupuestal incorporando la noción de planeamiento estratégico. Concebidos como herramientas de la transformación universitaria se plantean los siguientes objetivos:

- Responder a la demanda creciente por enseñanza superior, promoviendo la equidad social y geográfica y mejorando la calidad de la oferta pública.

- Impulsar la creación científica, tecnológica y artística estimulando su calidad.

- Promover la utilidad social del conocimiento contribuyendo a la solución de los problemas que hacen a la mejora de la calidad de vida de la población.

- Impulsar procesos de modernización de la gestión en condiciones de sustentar eficientemente las transformaciones de la Universidad de la República.

- Mejorar la atención de la salud que se brinda en el Hospital de Clínicas y con ello la calidad de la formación de los recursos humanos para la salud.

Para dar cumplimiento a estos objetivos estratégicos la UR define una batería de Proyectos Institucionales (P.I.) relacionados con las funciones de enseñanza, investigación, extensión y gestión y los procesos de descentralización universitaria. Dichos Proyectos impactan áreas diversas de la trama institucional y están dotados de una importante asignación presupuestal.

Con una radicación en distintas Unidades Ejecutoras, son gestionados centralmente de forma de asegurar una distribución equilibrada de estos recursos en virtud de necesidades comprobadas de los distintos servicios universitarios, derivados de sus respectivos planes estratégicos de desarrollo institucional.

Las modalidades de asignación de los recursos son diversas: concursos abiertos, asignación de acuerdo a criterios centrales, apoyo a actividades en ejecución sin financiamiento, entre otras. Cada asignación de rubros está entonces vinculada a un proyecto sustentable. Asimismo, es responsabilidad del servicio que recibe los rubros, redactar un Contrato Programa donde se describen con exactitud los objetivos las acciones a cumplir, los plazos de ejecución y los resultados esperados.

De esta manera, a partir de estas decisiones, es posible el logro de una distribución de recursos racional y vinculada a proyectos de desarrollo institucional. 
Se comienza a superar de esta manera, la modalidad de distribución incremental del presupuesto sobre la base de criterios históricos. Esta estrategia se acompaña de una adecuada política de información y seguimiento, cuyas informaciones están disponibles y accesibles en los medios de difusión de la U.R. por lo que se cumple también con un objetivo central, definido por el gobierno universitario, de responsabilidad social. Se trata de que la sociedad en su conjunto, pueda apreciar el grado de cumplimiento de los objetivos propuestos así como la forma en que se ejecutan los rubros provenientes del Estado.

\section{Segunda Reforma: la transformación universitaria actual.}

Según Landinelli (2007) "el proceso de la U.R. en las últimas dos décadas ha estado pautado por la voluntad de cambio". Y agrega: "En un arraigado entorno cultural de fidelidad a los principios de la autonomía y el cogobierno, a los valores discursivos y simbólicos afincados en su trayectoria y marcados en su ordenamiento legal, la institución ha buscado diseñar e implementar respuestas concretas a las exigencias de transformación que se han volcado sobre ella" (Landinelli, 2007: 73)

A continuación presentaremos la línea actuales de transformación universitaria, que ejemplifican una vocación institucional de avanzar hacia un verdadero "país de aprendizaje" (Arocena,2007) en consonancia con las demandas derivadas de una sociedad que reclama soluciones y un país que avanza hacia un modelo de desarrollo sustentable.

La actual agenda de transformaciones - jalonado por resoluciones del máximo órgano de gobierno universitario-, informa de una voluntad política de promover cambios sustantivos en la institución en la búsqueda de alternativas y soluciones innovadoras y socialmente pertinentes.

Los postulados principales pueden resumirse en los siguientes:

a) Vigencia plena de los principios de gratuidad, autonomía, cogobierno y compromiso social

b) Generalización de la enseñanza avanzada (diversificación, flexibilidad y descentralización de la oferta)

c) Integración institucional y académica (ampliación de la democracia y profundización de las medidas institucionales con el objetivo de contribuir a una mejor inserción de la educación superior en la totalidad del sistema educativo nacional) 
En documento relacionado con la reforma universitaria, aprobado por el Consejo Directivo Central extraordinario de fecha 30 de marzo de 2007, se señalaba que "La transformación del conjunto de la educación uruguaya debe estar orientada por el propósito de avanzar hacia la generalización de la enseñanza avanzada, de calidad y conectada con el trabajo a lo largo de toda la vida activa. Abrir a la mayoría de los jóvenes posibilidades de ese tipo tiene hoy tanta importancia para el Uruguay como la tuvo ayer la reforma varelia$\mathrm{na}^{14}$. Es clave tanto para el desarrollo integral del país como para disminuir la desigualdad entre regiones y entre sectores sociales" (U.R., 2007:13-14)

Bajo el supuesto que la actual reforma retoma el ideal latinoamericano plasmado en la Reforma de Córdoba, se impulsan en la actualidad medidas que posibiliten la constitución de un marco institucional que promueva el debate amplio y responsable de todos los actores involucrados. La creación del Comité de Transformación Universitaria, que cuenta con el respaldo de un equipo académico, la realización de ciclos de debate, el clima generalizado de participación, informa de una necesidad acuciante de cambios que posibiliten la ampliación de oportunidades educativas.

\begin{abstract}
"La generalización de la enseñanza avanzada, de calidad y conectada con el trabajo a lo largo de toda la vida activa requiere imaginación y audacia, grandes esfuerzos colectivos, y sustanciales recursos materiales. Tales recursos son necesarios para fortalecer y transformar la U.R., impulsando la educación terciaria en el interior, expandir la enseñanza tecnológica y mejorar sustantivamente la formación docente. Estas tareas educativas, y varias otras, requieren y justifican una gran inversión en el futuro nacional". (U.R., 2007:30)
\end{abstract}

Los ejes temáticos para pensar la transformación giran alrededor de los siguientes tópicos:

\title{
1. La construcción de un Sistema Nacional de Educación;
}

2. Definición de criterios para avanzar hacia un sistema de educación terciaria: flexibilización y diversificación de la enseñanza terciaria, carreras conjuntas con ANEP, creación de nuevas instituciones, programas compartidos, instancias de coordinación.

14 José Pedro Varela (1845-1879) impulsa una Reforma Escolar que sienta las bases del actual sistema de educación pública de Uruguay, definiendo el concepto de "Educación Popular" sustentado en los principios de laicidad, gratuidad y obligatoridad, con el objetivo político de formación de una ciudadanía responsable. 
3. Formación de docentes en coordinación con la ANEP

4. Programas Regionales de enseñanza avanzada para todo el país

5. Alternativas a los procesos de "desvinculación estudiantil"

6. Promoción de la investigación, su promoción, la difusión de sus resultados y de sus aportes comprobados o potenciales a la solución de los problemas sociales, su conexión con el desarrollo nacional.

7. La "extensión de la extensión", diversificación de las actividades en el medio, aportes a la renovación de la enseñanza;

8. Revitalización y ampliación del co-gobierno como objetivo mayor de la nueva reforma; transformaciones asociadas con la estructura de decisión;

9. Transformación y modernización de la estructura académica de la U.R, con atención prioritaria a las actividades interdisciplinarias;

10. La reforma y la gestión de la institución; eficiencia y fomento de la innovación institucional desde la participación. (U.R.,2007:36-37)

Para ello se han adoptado un número considerable de decisiones, que se encuentran en plena implementación, que coadyuvan al cumplimiento de las metas propuestas, A grandes rasgos, las transformaciones en marcha, giran alrededor de los siguientes ejes:

1. Generalización de la enseñanza avanzada y permanente. Sistema de educación flexible que asegure movilidad horizontal y vertical a estudiantes, docentes y egresados del sistema terciario que deseen continuar formaciones más largas; certificaciones que acrediten un primer nivel de estudios, acreditación de actividades de formación, incorporación de un sistema de créditos, creación de Ciclos Iniciales Optativos, diversificación de modalidades de cursado, generalización del uso de Tecnologías de la Información y la Comunicación (TIC), entre otras

2. Diversificación de los perfiles de formación, en consonancia con las demandas del desarrollo regionales y nacional; creación de nuevas instituciones públicas de carácter tecnológico terciario, laicas, gratuitas, autónomas, co-gobernadas y distribuidas en el territorio nacional. Definición de Programas Regionales de Enseñanza Terciaria (PRET) en coordinación con otras instituciones y la sociedad civil. 
3. Cambios en la estructura académica. Conformación de un espacio interdisciplinario de la UR que encare las tres funciones universitarias (enseñanza, extensión, investigación),

4. Promoción de la extensión y la vinculación entre las tres funciones universitarias. Programas Integrales para la resolución de problemas emergentes de la realidad y la búsqueda de alternativas conjuntas para el logro de una mejora de la calidad de vida de la comunidad. Investigación que priorice la contribución al desarrollo integral

Podemos afirmar en consecuencia que en el momento actual la UR se encuentra abocada a la realización de cambios y transformaciones guiada por el principio de democratización del conocimiento. En palabras de su actual Rector el Dr. Rodrigo Arocena: “ (...) la transformación educativa debe pensarse en el marco de la lucha contra la desigualdad y por la profundización de la democracia" (Arocena,2007:50)

Y agrega: "El conocimiento ha sido siempre una fuente de poder social, tanto del poder de unos grupos humanos sobre otros y sobre la naturaleza como de las diferencias de poder al interior de un mismo grupo. Ello es bien conocido. También es evidente que las dimensiones y las consecuencias del fenómeno se han multiplicado, adquiriendo en las últimas décadas un carácter realmente explosivo. La generación y utilización de nuevos conocimientos se han convertido en factores mayores de desestabilización y transformación de las relaciones entre la gente, trastocando hábitos y costumbres, suscitando tanto grandes oportunidades como amenazas, plateando dilemas hasta hace poco inimaginables, incidiendo de manera cada vez más aguda en las condiciones de vida y en la estratificación social. Asistimos así a la emergencia de una "sociedad del conocimiento"que tiene lugar por cierto de manera altamente asimétrica, sumando nuevas desigualdades a otras de viejo cuño" (Arocena: 2007,50-51)

En síntesis, los rasgos dominantes de esta reforma pueden resumirse de la siguiente manera: cambio y profundización de la democracia, con un fuerte sustento en los principios históricos que ha caracterizado el accionar de la U.R. desde hace más de 150 años.

Con una mirada prospectiva, es posible afirmar que esta Universidad se ubica dentro de las tendencias emergentes en América Latina que se plantean la meta de contribuir al desarrollo y mayor bienestar de la población, generalizando el acceso al conocimiento y conectando su generación y uso con las políticas sociales. 


\section{La actual agenda de la transformación: ejes y desafíos}

La segunda reforma de la UR, retoma los principios históricos de autonomía institucional y cogobierno, se guía por el ideal de profundización de la democracia institucional y se propone la generalización de una enseñanza avanzada, permanente, de calidad y combinada con el trabajo a lo largo de toda la vida.

Se propone, por lo tanto, contribuir a un nuevo desarrollo, de carácter integral y sostenible. Esta reforma apunta a la flexibilización de la enseñanza universitaria, ofreciendo perfiles y modalidades de formación diversificadas, en coordinación con otros niveles de la enseñanza pública, de modo de conformar un sistema articulado de formación terciaria, hoy inexistente.

La definiciones plasmadas en el Congreso de la Educación realizado en diciembre del año 2006 -verdadera instancia de debate nacional- posibilitó apreciar la necesidad de buscar formas organizacionales innovadoras y articuladas, que permitan una verdadera coordinación Interinstitucional. El país vive en la actualidad un clima verdaderamente fermental y fructífero en un contexto de discusión de una futura Ley de Educación. La U.R. no se encuentra al margen de este debate. La decisión de profundizar los cambios con criterio inclusivo, democrático y participativo, está en línea con la construcción de una "nueva institucionalidad" en convergencia con las actuales demandas.

Para ello, desde posturas profundamente innovadoras, se propone superar inadecuaciones y rezagos institucionales impulsando cambios radicales en la matriz institucional:

Destacamos en este sentido tres de ellos que a nuestro entender resultan buenos ejemplos de lo que venimos sosteniendo con respecto a la reforma universitaria en curso:

1. La puesta en marcha de un Programa Integral- uno de los objetivos relevantes del actual Plan Estratégico de Desarrollo de la U.R.-, con enfoque territorial y en red, que se propone dar un fuerte impulso a los procesos de enseñanza y aprendizaje inclusivos y sustentados en una ética de la autonomía, la participación y el diálogo. Con un estilo de gestión participativo, apoyado en estructuras flexibles, el programa integral se propone incluir a la población en la toma de decisiones a lo largo de todo el proceso. "La Universidad es de todos, no sólo de los universitarios y por tanto, todos tienen el derecho a aportar y opinar acerca de cualquier aspecto involucrado en el desarrollo de un programa integral, inclusive en su gestión" 
2. Busca asimismo, la promoción de un estilo de trabajo que habilite un intercambio entre saber académicos y populares, favoreciendo el desarrollo de nuevas formas de enseñar y aprender, investigar y producir conocimiento en contacto con la realidad. Y por último. Se busca potenciar la articulación de funciones y el enfoque interdisciplinario, reformulando los procesos de enseñanza y aprendizaje universitarios, con una perspectiva innovadora y flexible.

\section{La creación de Programas Regionales de Educación Terciaria (PRET)} invitando a colaborar en ellos a todos los actores sociales involucrados, potenciando el trabajo en la región y en consonancia con las demandas de formación derivadas del desarrollo social y productivo de la región. Estos Programas se asientan en cada una de las Sedes Universitarias del Interior del país y buscan construir una oferta académicamente pertinente, y fortalecer los lazos Inter.institucionales.

4. Impulso a la flexibilidad institucional y la innovación crítica, de modo de proceder a una profunda renovación de las formas tradicionales de construcción de las trayectorias estudiantiles y de los procesos de enseñanza y aprendizaje universitarios. Para ello se propone la modificación de la actual estructura académica - poco propensa al trabajo cooperativo y la interdisciplina- incorporando una estructura acoplada a las existentes, denominado espacio interdisciplinario, con el objetivo de articular esfuerzos hoy dispersos y aprovechar los avances logrados en el ámbito de las Redes Temáticas. En segundo lugar, se busca facilitar los tránsitos estudiantiles, modificando la organización de los estudios e incorporando la figura del "tutor-estudiante", hoy inexistente en la U.R.

La U.R se ubica en este escenario, junto a las universidades integrales y complejas que, como lo señala Landinelli (2007) "eludiendo situaciones de reflujo y desencanto, han intentado asumir procesos de modernización destinados a superar obsolescencias, perfeccionando los alcances de su misión pública, buscando responder desde el compromiso democrático a los desafíos planteados por la participación en la sociedad del conocimiento" (landinelli, 2007:69)

Preservando los valores históricos que constituyen su perfil identitario y su principal justificación, la U.R. avanza de forma sostenida hacia una reforma innovadora y pro-activa, definiendo prioridades y líneas de acción pautadas por el cambio y la búsqueda de una oferta universitaria pertinente y relevante del punto de vista social.

Dado que se trata de una universidad compleja, que no se plantea modificar sino reafirmar el criterio de acceso irrestricto, se propone el desarrollo de una 
verdadero proyecto académico, cuyo principal desafío es que logre concitar el máximo número de adhesiones y consensos. Su potencial de desarrollo -que nadie discute- se encuentra articulado a la también indiscutible, necesidad de debatir acerca de su viabilidad y pertinencia.

El debate acerca de los ejes de la trasformación actualmente promovido desde los órganos de gobierno y otras instancias centrales, posibilitará sin duda la implementación de las decisiones ya adoptadas y la incorporación de otras cuestiones hoy inexistentes. Se trata sobre todo, de promover la recuperación de la "confianza académica", y la sustitución de un estilo de organización institucional y trabajo académico caracterizado por la segmentación.

Se requiere por último, atender las resistencias instaladas en aquellos colectivos poco permeables a los cambios y la excesiva focalización en aspectos puntuales sin una adecuada contextualización.

Por último, corresponde insistir en la necesidad de implementar políticas que reconozcan el carácter heterogéneo de la institución, superando con ello la tendencia a aplicar políticas "unitalla". El reconocimiento de la existencia de culturas y prácticas académicas disímiles que coexisten y confrontan, debería ser el sustento de las necesarias instancias permanentes de seguimiento de los impactos educativos logrados, aplicando el criterio de responsabilidad y pertinencia social.

\section{Bibliografía consultada}

ARDAO,A (1950) "La Universidad de Montevideo. Su Evolución Histórica". Revista del Centro de Estudiantes de Derecho, No 68. UR, Montevideo.

ARDAO,A. (1968) Espiritualismo y positivismo en el Uruguay. UR, Colección Historia y Cultura. Montevideo.

ARDAO,A. (1985) "Carta de Arturo Ardao al secretariado de ASCEEPFEUU" Cuadernos de Política universitaria. Año 1, Nro 0, agosto de 1985. CIPE-ASCEEP-FEUU, Montevideo.

AROCENA, R. (2007) "Epílogo Provisional (Setiembre 2007): hacia la nueva ley de Educación". En: Hacia la reforma universitaria UR, Montevideo.

AROCENA,R.;SUTZ,J.(2001) La Universidad Latinoamericana del futuro. Tendencias-Escenarios-Alternativas. UDUAL, México.

BARROW, C. (1996) "The Strategy of Selective Excellence:redesigning higher education for global competition in a postindustrial society". En: Higher Education. 41: 447-469.Kluwer Academic Publishers, Netherlands. 
BENTANCUR DÍAZ,J;PARIS,B.(1995) Historia de la Universidad Ubicación: http://www.rau.edu.uy/universidad/uni_hist.htm ,Montevideo.

FEUU. VI CONVENCIÓN (1997) “Calidad Académica” En: UR: La Universidad de la República en un tiempo de cambio. UR, Montevideo.

GUARGA, R. (2007) "La construcción de la pertinencia de la Educación Superior”. En: VI Cumbre de Rectores de Universidades Públicas. (2007) 6 y 7 de julio de 2006. U.R.-AUGM-Junta de Extremadura., Montevideo.

LANDINELLI, J. (2007) "Las politicas de educación superior ante los desafios de la sociedad del conocimiento". En: BENTANCUR,N.(Coord.) (2007) Las políticas educativas en Uruguay. Perspectivas académicas y compromisos políticos. MEC-ICP-UR. Montevideo.

MINISTERIO DE EDUCACIÓN Y CULTURA (2007) Anuario estadístico de Educación 2006._MEC, Montevideo.

MINISTERIO DE EDUCACIÓN Y CULTURA (1997) Ordenamiento del Sistema de Enseñanza Terciaria Privada. Decreto Nro. 308/995._MEC, Montevideo.

PARIS, B. Breve historia de la Universidad de la República_UR, Montevideo.

PARIS, B. (1987) Autonomía de la Universidad uruguaya Ponencia presentada en el simposio sobre "La problemática actual de la Universidad latinoamericana” realizado en la UNAM, México, 22 al 25 de junio de 1987.(Publicación interna de la UNAM)

UR (2007) Hacia la reforma universitaria._UR. Montevideo

UR (2007) Carreras Universitarias._UR. Montevideo

UR (2005) Plan estratégico de la UR (PLEDUR) Documentos de trabajo del Rectorado Nro. 27 UR. Montevideo.

UR (2001) Plan estratégico de la UR (PLEDUR) Documentos de trabajo del Rectorado Nro. 10.UR, Montevideo.

UR (2001) Memoria de la Universidad 2000-2001. Documentos de Trabajo del Rectorado Nro.14. UR, Montevideo

UR (2000) Censo 2000. Principales características de los estudiantes de la Universidad de la República en 1999.Documentos de trabajo del Rectorado Nro. 7.Montevideo, setiembre de 2000, Montevideo. 